ÉGYPTE monde arabe

\section{Égypte/Monde arabe}

14 | 1993

Dits et écrits, mémoires et rites

\title{
Activités et pratiques enfantines autour du quartier du Khan al-Khalili
}

\section{Anna Madœuf}

\section{(2) OpenEdition}

\section{Journals}

Édition électronique

URL : https://journals.openedition.org/ema/577

DOI : 10.4000/ema.577

ISSN : 2090-7273

\section{Éditeur}

CEDEJ - Centre d'études et de documentation économiques juridiques et sociales

\section{Édition imprimée}

Date de publication : 30 juin 1993

Pagination : 113-132

ISSN : 1110-5097

\section{Référence électronique}

Anna Madœuf, «Activités et pratiques enfantines autour du quartier du Khan al-Khalili », Égypte/

Monde arabe [En ligne], 14 | 1993, mis en ligne le 08 juillet 2008, consulté le 07 juillet 2022. URL : http:// journals.openedition.org/ema/577 ; DOI : https://doi.org/10.4000/ema.577

Ce document a été généré automatiquement le 7 juillet 2022.

Tous droits réservés 


\title{
Activités et pratiques enfantines autour du quartier du Khan al- Khalili
}

\author{
Anna Madœuf
}

1 Au Caire, la présence des enfants est manifeste, attestée par leur importance numérique (presque un tiers des habitants a moins de douze ans). Ce sont, qu'ils exercent ou non une activité professionnelle, des acteurs urbains à part entière. C'est tout particulièrement dans la vieille ville, dont la morphologie se prête à leurs évolutions et où les pratiques en usage le leur permettent, qu'ils investissent l'espace public et sont partie prenante de la vie de quartier. Ils pratiquent, comme leurs aînés, l'art de la dérision; à l'aise sur leur territoire, ils tirent parti de chaque situation pour en être acteurs, pour s'y impliquer.

Dans les quartiers anciens, c'est par étapes que s'élabore l'apprentissage enfantin de l'extérieur-ville. Jusqu'à un an ils restent à la maison, hormis les sorties en famille, principalement avec les membres féminins, mères et sœurs ${ }^{1}$. A partir de deux ans, leur mobilité leur ouvre le champ des parties communes de l'immeuble : coursives, paliers, escaliers... Ensuite ils auront accès à l'impasse ${ }^{2}$ qui, qualifiée par l'usage de semi-privée au regard de son statut d'extension du logement, est le domaine privilégié des enfants de trois à dix ans. Là, soumis au contrôle social du voisinage dont ils sont - note JeanCharles Depaule ${ }^{3}$ - « à la fois sujets et agents », ils prennent leurs premiers contacts avec la ville tout en bénéficiant de la sécurité d'un espace familier et d'une gestion collective. C'est également dans ce cadre qu'ils vont s'initier à certaines formes de socialisation. On appartient à une impasse et en fonction de cette appartenance, des territoires et des clans se forment. L'entrée à l'école - dans ces quartiers il s'agit en général de l'école gouvernementale la plus proche, où l'on se rend à pied -, va considérablement élargir le champ des sorties et des découvertes urbaines; le statut d'écolier - qu'attestent uniforme et cartable - implique un droit de présence accru dans la rue et une plus grande liberté de circulation. 
3 En observant les cheminements des jeunes enfants, on s'aperçoit que très rares sont ceux qui se trouvent hors des parcours établis (souvent celui qui mène à l'école) ou loin de chez eux. Il existe de nombreuses limites et frontières symboliques très nettes, que les enfants ne franchissent pas.

$4 \quad$ L'objet de cette étude est d'observer, à l'intérieur d'un espace inclus dans la vieille ville - le Khan al-Khalîlî - mais marginal et autre que celui de leur cadre de vie habituel, les diverses pratiques et activités enfantines. Dans ce quartier, les enfants sont nombreux et leur présence revêt un caractère original ; leurs pratiques y apparaissent différentes de celles observables dans les zones à vocation majeure d'habitat. De plus, le fait qu'ils soient là, dans un secteur non résidentiel, implique évidemment qu'ils y sont venus, qu'ils ont effectué des déplacements motivés, importants à leur échelle. De même, hors du champ du contrôle familial et de leur territoire, leurs comportements peuvent se révéler particuliers.

5 A partir du constat d'une présence enfantine importante et en partie exogène, nombre de questions se posent : qui sont les enfants présents sur le terrain, d'où viennent-ils, quelles activités spécifiques ont-ils dans ce quartier, comment en investissent-ils les espaces, comment se les approprient-ils et s'organisent-ils, enfin quelles sont leurs perceptions des lieux?

6 Par ailleurs, vis-à-vis des adultes, quelles réactions suscite leur présence, comment sont-ils contrôlés, quelles sont les limites territoriales et/ou comportementales qui leur sont imparties, comment s'exerce la tolérance ou la non-tolérance à leur égard?

7 Les éléments de réponse découlent d'une enquête effectuée entre mars et juin 1992 dans le quartier du Khan al-Khalîlî et ses proches environs, enquête basée sur des observations assorties de questions non systématiques et sur de nombreuses « filatures » d'enfants, seuls ou en famille. L'étude concerne une cinquantaine d'enfants âgés de deux à douze ans environ, garçons et filles. Comme il ne s'agit pas d'un secteur résidentiel, les plus jeunes en sont pratiquement absents. La plupart des enfants recensés y exercent une activité, sédentaire ou ambulante; les autres viennent s'y promener ou s'y amuser. De ce fait, les enfants de plus de six ans, âge à partir duquel ils travaillent ou déambulent seuls, sont surreprésentés. De plus, la population masculine est nettement majoritaire puisque, d'une part, les filles ne travaillent pas dans les secteurs d'activités artisanales présents dans cet espace et, d'autre part, elles sont soumises - comme partout - à davantage d'interdits spatiaux que les garçons. Elles fréquentent moins les rues, cette restriction s'accroissant dès qu'elles atteignent une huitaine d'années. Aussi, paradoxalement, les enfants les plus jeunes présents sur cet espace sont des fillettes d'environ cinq ans.

8 Les observations ont été menées à différentes heures en journée et en soirée, à partir de postes précis (cafés du Khan al-Khalîlî, divers points de la place al-Husayn, alentours du souterrain et de la passerelle traversant la rue al-Azhar...). Les passages des enfants ainsi que la fréquence et la répétition de ces passages, les allures des enfants et leurs attitudes, leurs accessoires, leurs relations, leurs échanges avec les adultes..., tous ces éléments ont été relevés.

9 Lors d'enquêtes centrées sur un public enfantin, on relève des difficultés de plusieurs types. Les enfants, lorsqu'ils sont questionnés, ont tendance à apporter des réponses conventionnelles et essayent d'exprimer ce qu'ils croient être la réponse attendue ${ }^{4}$. On ne peut non plus leur poser trop de questions à la suite, car ils se lassent vite. Ils ont 
souvent des perceptions vagues de l'espace et du temps, qui peuvent varier nettement d'un individu à l'autre, et des difficultés à formuler les motivations de leurs actes.

10 Aussi faut-il, pour vérifier certains éléments de réponse, recourir aux adultes, chez lesquels on rencontrera d'autres types de réticences : par exemple, pour justifier le travail des enfants, des liens familiaux sont souvent mis en avant (liens qui n'existent pas toujours, même s'il est d'usage de dire que l'on forme une « famille de travail »).

11 Il est de surcroît difficile de suivre plusieurs jours durant les enfants de passage, marchands ambulants ou désœuvrés. Comment savoir quand ils reviendront, à quelle heure précisément, et où ils se tiendront...

12 Ces observations doivent être nuancées par le fait que chaque enfant est le produit d'une classe sociale, fait déterminant en ce qui concerne les observations de microphénomènes vécus et/ou générés par des « petits d'hommes ».

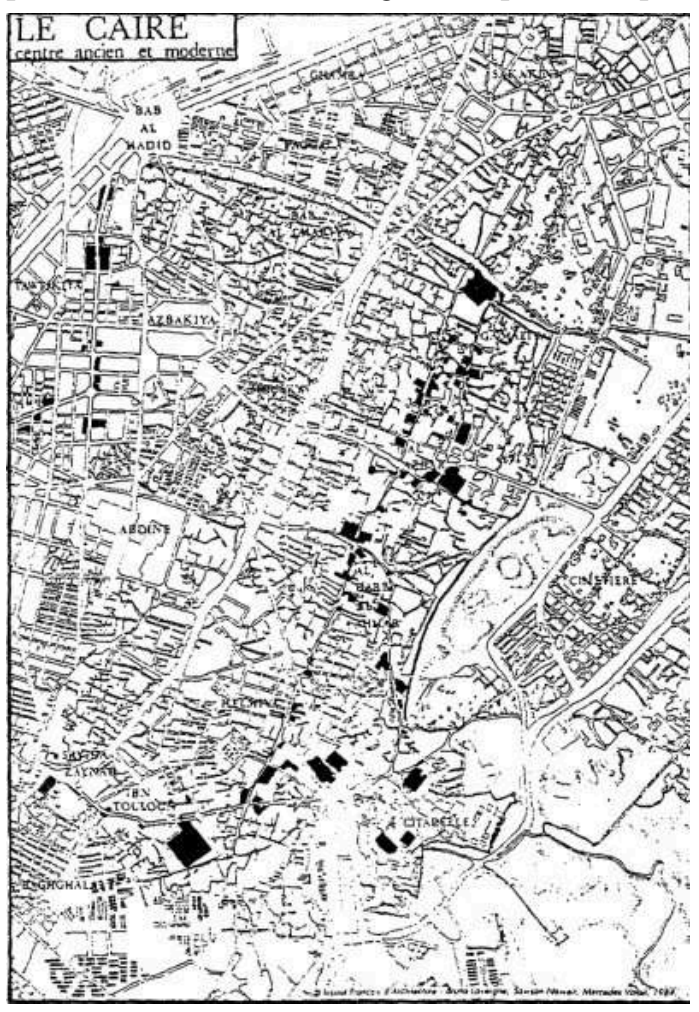

Le Khan al-Khalîlî, un " centre-ville »

II ne s'agit pas dans ces lignes de décrire un espace qui a déjà fait l'objet de plusieurs travaux - notamment ceux de Jacques Berque ${ }^{5}$, mais, en premier lieu, de rappeler la diversité des types d'espaces et paysages urbains en fonction d'un acteur principal, l'enfant.

Le Khan al-Khalîii et ses alentours immédiats forment, par rapport au reste de la vieille ville, un ensemble différencié où abondent interférences et brouillages dus à l'importance de la fréquentation extérieure, ensemble essentiellement voué - en parallèle et en complément de sa fonction d'espace cultuel - au commerce et à l'artisanat. Il peut être perçu comme un bazar pour touristes, un centre commercial indépendant et déconnecté de son cadre, de son milieu d'ancrage - la vieille ville - et apparaitre de ce fait comme un espace extra-territorialisé. 

quartiers de la ville les plus difficiles à catégoriser ; bénéficiant d'un statut particulier aux yeux des Cairotes, il est qualifié à la fois d'« historique ", de "touristique » et de cha'bi (" populaire»). Au centre géographique de la vieille ville, à la croisée des seuls axes importants qui la desservent et l'irriguent (rues al-Azhar, al-Mu'izz et al-Muski), il se situe au cœur de zones d'activités artisanales multiples (verrerie, teinturerie, textile, travail des métaux et du cuir...). Ces quartiers mitoyens, vassaux du Khan, le fournissent en produits divers destinés à la vente locale ou à l'exportation (essentiellement vers les autres villes touristiques d'Égypte). Leïla Ammar et Philippe Panerai soulignent « la vigueur de la centralité urbaine » autour de plusieurs quartiers, dont celui du Khan al-Khalîlî.

Par ailleurs, la multiplicité des lieux de culte, de pèlerinages et de célébrations religieuses (dont le populaire Mawlid al-Nabi, Nativité du Prophète) confèrent à cet espace une aura prestigieuse et sacralisée.

Le Khan et les grandes mosquées d'al-Azhar et d'al-Husayn, éléments attractifs sur une zone d'influence commune, ont permis, malgré leurs fonctions antithétiques, la cohésion de ce quartier. Dans la trilogie de Naguib Mahfouz', al-Husayn et le Khan apparaissent comme deux quartiers très distincts; or, d'après l'enquête réalisée en 1989 par Leïla Ammar et Mona Charara ${ }^{10}$, il semble qu'une perception commune de ces secteurs se soit imposée tant aux habitants de la vieille ville qu'aux autres Cairotes.

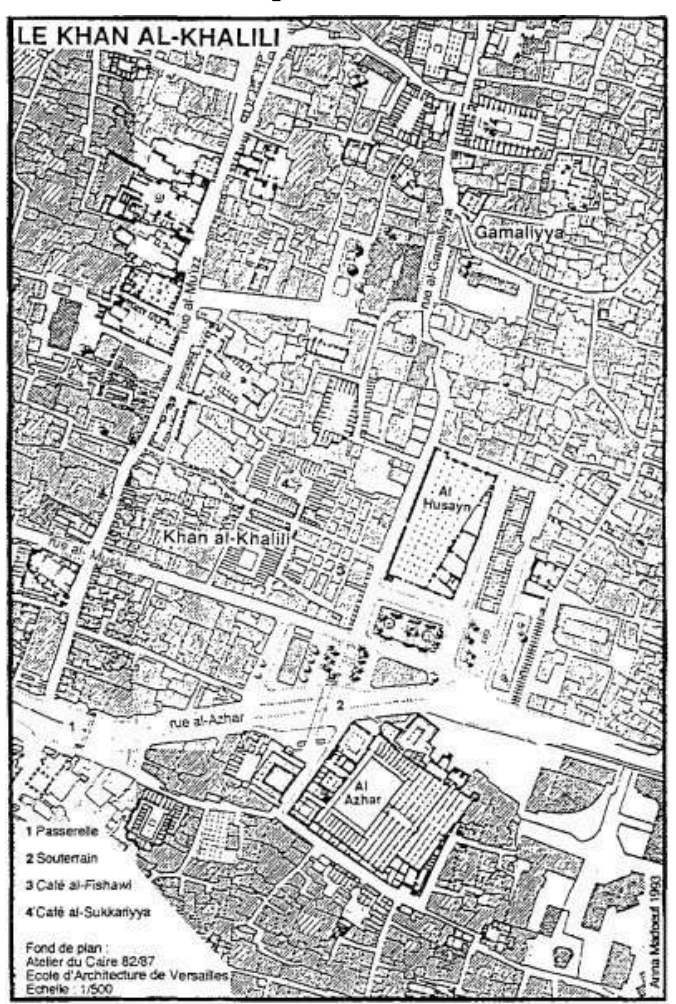

Espace clos, le Khan al-Khalîlî forme un quadrilatère compact aux accès peu nombreux : deux sur la rue al-Muski, deux sur la rue al-Mu'izz et deux par la place al-Husayn (dont un étroit passage entre deux boutiques). $\mathrm{Si}$, au nord, d'autres accès mènent à la Gamaliyya, ils ne sont utilisés que par les habitants du quartier ou les fournisseurs. 

phénomène original, certains y viennent seuls, ou avec un ami, des quartiers périphériques mais parfois aussi de plus loin, par exemple du quartier spontané de Manchiat Nasr, au-delà de la nécropole de Qayt Bey. Ils viennent là précisément et restent dans ce secteur. Leurs motivations sont claires - soit ils y ont une activité marchande, soit ils s'y promènent - mais la destination finale est connue à l'avance. Ils évoluent facilement dans ce quartier aux points de repère multiples et variés, où ils peuvent se mêler à la foule. Ils utilisent ce lieu comme un centre-ville, un espace à la fois attractif et fixatif, où évoluent des gens marginaux par rapport au quartier et aux fonctions apparentes premières du lieu. Cet espace comporte en effet tous les éléments d'un centre-ville : le prestige culturel lié à la présence des monuments anciens et des grandes mosquées, les commerces, les espaces récréatifs et de sociabilité, l'animation, la foule... facteurs qui exercent une ou des attirances sur ces enfants selon le but de leur déplacement.

Rythmes de temps et d'espaces 
24 Les enfants ne sont pas soumis à des habitudes fixes, même si des constantes de fréquentation se font jour :

25 - ils viennent plus particulièrement en fin d'après-midi et en soirée, le jour le plus fréquenté étant le vendredi ;

26 - les vendeurs ambulants établissent des tournées et, de manière générale, les enfants ont leurs lieux de prédilection ;

27 - la durée de leur présence sur les lieux est inconstante et sujette à de nombreux facteurs (horaires scolaires s'ils sont scolarisés, vente plus ou moins rapide de leurs produits, ennui, lassitude...)

28 Les pratiques enfantines en ces lieux varient selon les rythmes temporels (heures, saisons, fêtes, vacances scolaires...). Cette caractéristique s'est révélée particulièrement pertinente sur cet espace où sont observables des différences de fréquentation et d'usage très importantes entre les périodes courantes et celles des fêtes, spécialement le Ramadan. Certains enfants viennent uniquement à cette époque, seuls ou en famille, pour les attractions et amusements propres à cette occasion (fête foraine rue al-Azhar, animations festives) ou pour vendre divers produits afin d'apporter à leur famille un complément financier, bienvenu au cours de ce mois.

29 Sont également notables d'importantes variations selon les heures; dans la journée sont présents les enfants qui travaillent, certains du matin jusqu'au soir. Dans le courant de l'après-midi arrivent les marchands. Certains vont à l'école le matin et viennent ensuite (ou inversement). Il en va de même pour ceux qui travaillent (Tawfiq Aclimandos $^{14}$ relevait qu'en 1982-83, environ $16 \%$ des enfants égyptiens n'entraient jamais à l'école et $8 \%$ la quittaient définitivement au cours du cycle primaire).

30 - Toujours en fin d'après-midi viennent jouer et se promener les enfants du voisinage et ceux qui accompagnent leurs parents pour des courses. En soirée arrivent des familles qui sortent en ville, pour dîner ou prendre une boisson, profiter de l'animation.

Les activités du Khan al-Khalîlî

31 Le travail des enfants au Caire - tout spécialement dans la vieille ville où les activités artisanales sont très dynamiques - est un phénomène important ; Gunter Meyer ${ }^{15}$ note que dans 80 ateliers de la Gamaliyya, étudiés en 1987, les garçons de moins de 15 ans représentent $20 \%$ des employés et ceux de 5 à 9 ans, $6 \%$; ces chiffres s'accroissent pour des secteurs nécessitant une main-d'œuvre nombreuse et peu qualifiée, comme celui de l'aluminium où la proportion d'enfants peut doubler - $12 \%$ de moins de 10 ans et $34 \%$ de moins de 15 ans.

32 Les fabriques et ateliers abondent dans le Khan, en particulier dans les cours et aux étages des wikâla (caravansérails), et emploient des enfants placés en apprentissage presque exclusivement des enfants de la vieille ville, qui ont parfois un lien de parenté avec les artisans ou employés, ou sont placés par leur père, propriétaire d'un atelier dans la vieille ville. L'apprentissage de l'artisanat et du commerce au Khan est réputé. Ces enfants-ouvriers y apprennent ou exercent un métier, travaillent les métaux (cuivre, aluminium, métaux précieux), s'initient à l'orfèvrerie et à la marqueterie.

33 Les boutiques et ateliers emploient aussi des enfants-commis chargés de l'entretien ou des divers services liés à la vente (polissage et empaquetage des objets) et à la vie quotidienne, remplissant également les tâches de coursiers et de portefaix. On compte en moyenne un enfant pour trois boutiques, certains travaillant pour plusieurs 
commerçants qui s'arrangent pour la répartition des tâches et la rétribution. Leurs constantes allées et venues sont observables depuis certains endroits stratégiques, notamment le Café Fichawi qui commande l'accès à un passage où se tient une boutique de nourriture à emporter. On y observe en permanence une cohue d'enfants portant des sandwiches de fûl-ta 'meyya (fèves et beignets) et des plateaux de verres de thé. Les définitions d'apprenti et de commis sont floues - certains sont les deux à la fois mais de manière générale, ce sont les enfants - quelle que soit leur fonction exacte qui font les courses et se déplacent dès que cela s'avère nécessaire; c'est également à eux qu'incombe la charge du thé. Mouvement et déplacement les caractérisent, ces fonctions étant proportionnellement inverses à l'âge, à l'autorité, à la respectabilité, à l'importance du statut social.

Les enfants chargés de ces tâches adoptent, lors de leurs déplacements, une allure affairée, une attitude assurée ; ils intériorisent leur rôle de travailleur, ont entre eux des rapports professionnels, se rassemblent pour partager leur repas et ne fraient pas avec les autres enfants, en particulier les vendeurs ambulants dont le statut est inférieur au leur.

Topographies des vendeurs ambulants

On peut estimer à environ une centaine (leur nombre exact est impossible à évaluer) les vendeurs ambulants exerçant sur cet espace. En moyenne sont présents simultanément une vingtaine d'enfants, mais ce chiffre est fluctuant.

Ces marchands ambulants commandités par des adultes - parents ou employeurs vendent à l'unité des objets de peu de valeur, peu encombrants et dont ils n'ont que quelques exemplaires. Leur panoplie marchande, spécifique, se compose essentiellement de paquets de mouchoirs en papier, allumettes, barrettes à cheveux, peignes, colliers de jasmin, porte-clés, cacahuètes, chewing-gums, etc... Aucun enfant ne vend de lunettes de soleil ni de portefeuille, objets très répandus mais exclusivement distribués par des adultes.

Bien que ne pratiquant pas la vente mais la mendicité, sont présents sur les mêmes parcours et aux environs des mosquées des enfants (en général des fillettes) accompagnant un adulte infirme.

Les parcours des « ambulants » sont établis en fonction de tournées incluant les cafés et restaurants de la rue al-Muski, les terrasses place al-Husayn et quelques lieux à l'intérieur du Khan al-Khalîli, mais situés en bordure, comme le Café Fichawi et celui du Hagg, qui sont mitoyens. Les plus audacieux font parfois des incursions jusqu'au Café Sukkariyya mais ne s'attardent pas dans ces ruelles. Certains endroits leur sont totalement interdits comme le récent Café Naguib Mahfouz (le plus touristique), d'où ils sont systématiquement chassés.

Pour les serveurs du Café Fichawi, par contre, leur présence est banale (haga 'âdiyya), et même si l'on n'y rencontre plus les jeunes ramasseurs de mégots évoqués par Cossery ${ }^{16}$, les vendeurs ambulants y affluent. La morphologie des lieux s'y prête (il s'agit d'un café-passage) de même que l'ancienneté du café et des pratiques qui y ont toujours cours.

41 Ils effectuent ainsi une tournée selon un parcours type en évitant de pénétrer à l'intérieur du Khan, malgré les détours que cette exclusion leur impose. Cette tournée d'une vingtaine de minutes environ peut être recommencée plusieurs fois de suite. Les 
enfants mettent toujours plus longtemps que les adultes qui exercent le même métier en empruntant le même trajet car ils s'attardent et se distraient davantage.

Dans le Khan al-Khalîlî proprement dit, leur présence est tolérée mais néanmoins strictement limitée dans le temps, l'espace et en fonction de chaque individu. Si la pratique de certains axes de circulation leur est concédée, en revanche, le stationnement devant les boutiques est prohibé : ils doivent rester en retrait. Durant leurs parcours, ils ne doivent ni s'arrêter, ni s'installer, non plus accoster les passants pour vendre leurs produits. Les étapes qui leur sont permises sont fixes. Sur leurs lieux de vente, ils ne doivent ni insister ni s'attarder. Ces codes ne sont pas toujours respectés et il y a intervention des adultes, gestionnaires des lieux, lorsque s'amorce une dérogation à ces règles tacites. Ainsi s'exerce la forme principale du contrôle social à leur égard.

43 Comment s'exprime la tolérance vis-à-vis de ceux qui ont le droit de pratiquer un métier ambulant? Les réponses sont particulières à chaque enfant et relèvent de plusieurs facteurs; tel enfant a gagné l'autorisation tacite d'être là "parce qu'on le connaît »; cette connaissance/reconnaissance a plusieurs sources : l'enfant a su gagner la sympathie, sa famille est connue, il a bénéficié de protections, il est du quartier... Il semble que les vendeurs ambulants du Khan soient des enfants du quartier, c'est-à-dire du proche périmètre pédestre, (Batniyya, al-'Utûf, Darrâsa, Gamaliyya...) ce qui est corroboré par leurs réponses à ce sujet.

44 . En aval de celle des commerçants (lorsque celle-ci s'avère insuffisante), est présente une autre forme de contrôle et de régulation, celle de la police, qui s'exerce de manière soutenue lorsqu'il y a afflux d'ambulants, notamment pendant le mois de Ramadan, la fréquentation de ces lieux s'accroissant considérablement. La police arrête les enfants et les retient de quelques heures à une journée; ils sont soumis à des amendes et s'ils ne bénéficient pas de la protection ou de l'intervention d'une personne influente (un commerçant), ils sont repris lors de la rafle suivante, mesure qui s'avère très dissuasive. Un des lieux où s'exerce cette détention est un poste situé dans le passage menant au Café Sukkariyya. Bien que les enfants soient ici « exposés» - visibles par tous car du fait de l'exiguïté du poste, les détenus sont gardés dans le passage -, ce type d'événement ne suscite pas de réaction particulière, les personnes présentes expliquant cette mesure par le fait que ce sont des awlâd al-châri' (enfants de la rue) dont le sort leur est indifférent.

45 En dehors du Khan al-Khalîlî, le long des rues qui le bordent et sur la place al-Husayn, se trouvent d'autres vendeurs ambulants qui viennent de plus loin, parfois d'au-delà de la vieille ville, et qui se tiennent dans ces secteurs parce que les commerçants des boutiques qui commandent l'accès au Khan les refoulent. Ils vendent les mêmes objets ainsi que des souvenirs (papyrus, chameaux en cuir, cartes postales) concurrentiels des produits du Khan et qu'il serait difficile d'écouler dans ce périmètre. Certains sont cireurs de chaussures, les cafés du Khan ayant leurs cireurs (adultes) attitrés. Dans ce secteur en marge, les parcours ne sont pas aussi définis que ceux du Khan, les contraintes y sont moindres, le contrôle des adultes plus diffus. Les enfants peuvent ici bénéficier de plus de mobilité spatiale alors que, paradoxalement, ils sont moins tolérés. Cependant, ils tiennent en général à s'attribuer des "postes " plus ou moins fixes et des territoires.

46 La pratique d'un espace implique toujours des habitudes, des cheminements, un marquage et, même si les limites, les règles et les interdits sont peu contraignants, 
chacun se crée son espace familier et ses habitudes, tout spécialement les enfants qui affectionnent les repères et investissent un champ spatial plus restreint que les adultes, parce qu'ils sont moins libres dans leurs investigations, mais aussi parce que leur imagination compense. Ainsi se créent des habitudes de sites, se dessinent des topographies enfantines déterminées par divers facteurs: un vendeur de papyrus rue al-Muski a choisi comme centre de sa zone d'influence une buvette parce que « la dame qui la tient est gentille »; un vendeur de barrettes se tient en général près de la station de taxis car il " aime regarder les voitures et les gens qui arrivent "; d'autres se postent aux débouchés de la passerelle et du souterrain qui traversent la rue al-Azhar car ici, la foule est dense, ce qui accroît la clientèle potentielle et forme aussi un écran rassurant.

D'autre part, celui qui s'octroie une zone doit l'affirmer en la défendant vis-à-vis d'autres enfants : ainsi se créent des rapports de force (les enfants étant eux-mêmes tributaires des adultes dans leurs attributions d'espaces). Comme tous les enfants ne sont pas présents en même temps, ces territoires se juxtaposent.

En parallèle, on observe une autre activité, celle des « rabatteurs » ou enfants affectés à une boutique - principalement les parfumeries de la rue al-Muski - qui arpentent une portion de rue pour ramener des clients ; mais ce métier est marginal et semble réservé à la parfumerie. De même, quelques enfants du quartier accostent et suivent les touristes, parfois sur un long trajet et durant plusieurs heures ; trop jeunes pour être guides, ils se contentent d'être accompagnateurs.

Pratique des sous-espaces

Les jours d'affluence, on note la présence insolite d'enfants apprêtés (surtout des fillettes) assis sagement sur de petites pelouses entourées de grillages, entre le débouché du passage souterrain et la rue al-Muski. Ces lieux ont en fait été "détournés » en un système de garderie très commode. Les mères déposent là leurs enfants avant d'aller faire les courses (la station de bus est très proche), elles leur laissent parfois de l'argent pour acheter une sucrerie ou une boisson, mais ils ne doivent pas sortir du parc. Souvent, des marchands viennent proposer, par dessus le grillage, des sirops ou des friandises. Les tenanciers des buvettes installées autour de ces enclos les surveillent d'un œil et en échange, en parlant, la famille prend une consommation sur place.

On retrouve la même interprétation de ces enclos devant la mosquée al-Azhar où sont installées des femmes exposant leur marchandise dans des paniers. Elles viennent avec leurs enfants - les plus grands vont vendre ces produits plus loin, les plus jeunes restent sur la pelouse clôturée à laquelle elles sont adossées. L'accès à la pelouse, en principe interdit, est toléré pour eux.

51 A proximité de ces lieux, sur la bordure est de la mosquée al-Husayn, sont implantés des magasins sous arcades devant lesquels se tiennent des étals serrés proposant des jouets : diverses répliques miniatures d'instruments de musique - percussions (tabla), luths décorés ('îd), clarinettes (mizmar), crécelles, cymbales - et autres jouets non musicaux (cerfs-volants, toupies, etc.). Ces jouets ne sont pas les mêmes que ceux exposés ailleurs, rue al-Muski par exemple : ces lieux sont fréquentés par les pèlerins et la tradition veut qu'ils achètent pour leurs enfants ce type de jouet. En retrait, une placette offre des animations destinées aux jeunes : attractions de forains (tir de ballon ou de cible, lancer de balles, pétards), vente de pop-corn, carrioles de masques et chapeaux de fête en carton. Ce lieu qui s'anime surtout en soirée est également fréquenté par les enfants du quartier venus seuls. Ici se tiennent les marchands de 
poupées et sujets en sucre confectionnés lors des fêtes de saints (mawlid). A proximité, sur la bordure orientale de la place al-Husayn, s'expriment diverses formes d'activités ludiques: de jeunes loueurs de bicyclettes y proposent des tours d'une vingtaine de minutes pour une livre; des enfants viennent y jouer car il y a de la place, de la tranquillité. S'ébauchent ici des jeux improvisés de course, de mouvements non installés, fugaces. Ces deux espaces complémentaires forment un lieu particulier de sociabilité et de rendez-vous enfantins. Certains s'y rencontrent, y nouent des amitiés, éphémères ou durables, mais originales car elles ne sont pas le fruit de rencontres de voisinage, scolaires ou familiales. Certes, ces pratiques peuvent se compléter et se superposer. Les notions d'activité, de jeu, de promenade, "d'errance», ne sont pas figées et ne s'excluent pas.

L'enfant, un présent furtif

Le regard de l'acteur exogène, de l'étranger - qu'il soit touriste, pèlerin ou client cairote - est déterminant par rapport aux comportements adoptés et tolérés, spécialement vis-à-vis des enfants. De plus, le Khan al-Khalîlî est un petit espace cerné de quartiers polymorphes, cadres de pratiques liées à la vie quotidienne, lesquelles n'ont pas lieu d'être dans le secteur du Khan et doivent cesser dès son « seuil » franchi.

Aussi peut-il être éclairant de noter l'absence, en ces lieux, de certains phénomènes pourtant courants dans la vieille ville : sur cet espace n'existent ni bandes d'enfants ni groupes constitués, nul n'y joue au foot-ball alors que cette activité (très populaire au Caire) est un indice d'appropriation de l'espace : elle implique que l'on connaisse ses partenaires et que l'on soit sur son propre territoire, ou dans un espace familier. Rares sont les enfants du voisinage qui effectuent la démarche de venir jouer dans ce secteur. De nombreuses activités ludiques (jeux de ballon, marelle, etc.) et pratiques enfantines, notables ailleurs dans la vieille ville, n'ont pas cours ici. Les enfants ne s'approprient pas l'espace et par conséquent n'inscrivent aucune marque de leur présence. Sont absents ces lieux un peu secrets (terrains vagues, recoins tranquilles) qu'ils affectionnent, de même que les espaces marginaux qu'on leur cède et dont ils se réservent l'usage. Le Khan al-Khalîlî est une forteresse, et la place al-Husayn un parvis où ne peuvent être créés ou aménagés de sous-espaces plus intimes.

Les enfants ne sont ici que des passants, et à ce titre, ils ne peuvent ni investir ni s'approprier les lieux; les interdits y sont multiples, les tentatives d'appropriation refoulées. Soumise au bon vouloir des adultes, la présence enfantine est tolérée, en aucun cas encouragée. En découle une appropriation fugitive et une présence «à la sauvette ", non construite. Il s'agit d'un lieu de consommation, cette fonction niant la légitimité de leur présence hors du cadre familial.

L'évocation des enfants présents sur ce territoire amène à celle des enfants qui n'y sont pas, qui restent en marge, en retrait derrière des frontières matérielles mais surtout symboliques. Ce clivage est illustré par le passage souterrain, frontière pour ceux qui habitent l'autre côté de la rue al-Azhar, coupée en son centre de hautes grilles et parcourue d'un flot de véhicules. Depuis leur quartier, ces enfants aperçoivent un autre univers, auquel ils n'osent accéder. C'est le cas de plus petits qui jouent souvent dans ce souterrain et les escaliers qui y mènent, faisant de perpétuels allers-retours dans ce tunnel entre deux mondes. Ils ne franchissent pas le seuil, n'émergent pas côté alHusayn, car c'est mamnu' (interdit) et leur envie semble moins tenace que leur appréhension. Ainsi ils ne transgressent pas ces limites car ils ont intégré les interdits 
du contrôle familio-social et "se sentent » surveillés, même en l'absence d'adultes exerçant effectivement ce contrôle.

Quand ils seront plus grands, ils iront « là-bas » parce que cet espace entrevu, deviné, imaginé, excite les convoitises enfantines: "C'est beau, il y a beaucoup de gens et beaucoup de choses ».- l'abondance signifiant la qualité. Peut-être est-ce leur façon de percevoir et d'exprimer que «dans le quartier appauvri mais non déchu, la ferveur reste native, la beauté présente, la baraka diffuse ${ }^{17}$ ».

Cet article reprend les thèmes évoqués au cours d'une communication au séminaire "Maîtrise de l'espace» du CEDEJ en juin 1992, et entrant dans le cadre d'une thèse de géographie sur "Les rapports des enfants à l'espace dans la vieille ville du Caire " sous la direction de Jean-François Troin, URBAMA. Tours.

\section{NOTES}

1. Al-Messiri Nadim Nawal, « The Concept of the Hâra. A historical and sociological study of al-Sukkariyya », Annales Islamologiques, t. XV, Le Caire, IFAO, 1979.

2. Nous avons choisi d'utiliser le mot « impasse » comme terme générique pour désigner des types morphologiques divers (darb, 'atfa, zuqàq, hâra) caractérisés dans cette étude par leurs dimensions restreintes, mais qui ne sont pas nécessairement des voies sans issue, cf. Baker F., "Questions de toponymie ", Égypte/Monde Arabe n ${ }^{\circ} 5$, Le Caire, CEDEJ, 1991.

3. Depaule Jean-Charles, « Des territoires en formation. Jeunesse et urbanisation au Caire ", Égypte/Monde Arabe n 1, Le Caire, CEDEJ, 1991.

4. $c f$. Chombart de Lauwe Marie-José, « Un intérêt ambigu, des discours piégés », in Dans la ville, des enfants. Les 6-14 ans et le pouvoir adulte : enjeux, discours, pratiques quotidiennes. Autrement $\mathrm{n}^{\circ}$ 10, Paris, Le Seuil, 1977.

5. Berque Jacques, Al-Shakaa Mustafa, «La Gamàliya depuis un siècle. Essai d'histoire sociale d'un quartier du Caire ", Revue des Etudes Islamiques, XLII-1, Librairie orientaliste, 1974.

6. «Vieille ville » est le terme communément utilisé pour parler d'un espace pour lequel L. Ammar et P. Panerai envisagent l'appellation de "vieille ville moderne », en s'appuyant sur le fait que la majorité des édifices date de moins d'un siècle et au vu des transformations qui s'y réalisent. Cf. Ammar Leïla, Panerai Philippe, « Le Caire : observations sur le tissu urbain de la ville ancienne », Programme pluri-annuel 1986-1989, la constitution du territoire et la construction de la ville, fascicule $\mathrm{n}^{\circ} 18$, Laboratoire de recherche " Histoire architecturale el urbaine/sociétés ", Ecole d'Architecture de Versailles, 1991.

7. Labib Albert, Battain Tiziana, « Le Caire-mégalopole perçue par ses habitants », Égypte/Monde Arabe n`5, Le Caire, CEDEJ, 1991.

8. Ammar Leila, Panerai Philippe, op. cit., p. 70.

9. Mahfouz Naguib, Impasse des Deux Palais, 1985 ; Le Palais du désir, 1987 ; Le Jardin du passé, 1989, J.-C. Lattès, coll. « Lettres arabes ». 
10. Ammar Leila, Charara Mona, "A travers les hâra du Caire fatimide ", Égypte/Monde Arabe $\mathrm{n}^{\circ}$ 5, Le Caire, CEDEJ. 1991.

11. David Jean-Claude, «Centralités anciennes et actuelles dans « Al Qâhira » », à paraître in Etablissements de rapport au Caire aux périodes mamelouke, ottomane et contemporaine, Le Caire, IFAO.

12. Nahâssin: dinandiers, de nahâss : cuivre.

13. AI-'Utûf: pluriel de al-'atfa : l'impasse.

14. Aclimandos Tawfiq, "L'enseignement à l'étude ", Revue de la presse égyptienne, 27, Le Caire. CEDEJ, 1987.

15. Meyer Gunter, « Manufacturing in old quarters of central Cairo », Eléments sur les centres-villes dans le Monde arabe, fascicule de Recherches n²19, Tours, URBAMA, 1987. 16. Cossery Albert, Mendiants et orgueilleux, Paris, Terrain vague, 1990.

17. Berque Jacques, Al-Shakaa Mustafa, op. cit. p. 96.

INDEX

Mots-clés : enfants, Le Caire, travail, urbanisation

\section{AUTEUR}

ANNA MADEUF

CEDEJ/OUCC 